Pacific

Journal of

Mathematics

\title{
HYPONORMALITY OF BLOCK TOEPLITZ OPERATORS
}

CAXING Gu, Jacob Hendricks AND DANIEl RUtherford 


\title{
HYPONORMALITY OF BLOCK TOEPLITZ OPERATORS
}

\author{
CAXING Gu, JACOB HENDRICKS AND DANIEL RUTHERFORD
}

\begin{abstract}
We show that for a block Toeplitz operator $T_{G}$ to be hyponormal, there is a matrix analogue of Cowen's condition for a scalar hyponormal Toeplitz operator, but an additional condtion, $G^{*} G=G G^{*}$, is needed. A detailed analysis is given for the hypornormality of $T_{G}$ if $G$ is a matrix trigomometric polynomial or $G$ satisfies an extremal condition. Relevant results on kernels of block Hankel operators are also obtained.
\end{abstract}

\section{Introduction}

The block Toeplitz operator with matrix symbol $F \in L^{\infty}\left(\mathbb{C}^{n \times n}\right)$, denoted $T_{F}$, acts on the vector-valued Hardy space of the unit disk, $H^{2}\left(\mathbb{C}^{n}\right)$ ( $n$ is finite), and is defined by $T_{F} h=P(F h)$, where $P$ is the projection from $L^{2}\left(\mathbb{C}^{n}\right)$ to $H^{2}\left(\mathbb{C}^{n}\right)$. The block Hankel operator with the same symbol, $H_{F}: H^{2}\left(\mathbb{C}^{n}\right) \longmapsto H^{2}\left(\mathbb{C}^{n}\right)$ is defined by $H_{F} h=J(I-P) F h$, where $J$ denotes the unitary operator from $H^{2}\left(\mathbb{C}^{n}\right)^{\perp}$ to $H^{2}\left(\mathbb{C}^{n}\right)$ given by $J\left(e^{-i m \theta}\right)=e^{i(m-1) \theta}$ for $m \geq 1$. Notice that the shift operator $S$ equals $T_{z I_{n}}$, where $I_{n}$ is the $n \times n$ identity matrix.

In fact, a given bounded linear operator $T$ is a Toeplitz operator if and only if $S^{*} T S=T$, and a given bounded linear operator $H$ is a Hankel operator if and only if $H S=S^{*} H$. It can be readily verified that $T_{F}^{*}=T_{F^{*}}$, and letting $\widetilde{F}=F^{*}(\bar{z})$ we have $H_{F}^{*}=H_{\widetilde{F}}$. Let $G \in L^{\infty}\left(\mathbb{C}^{n \times n}\right)$. There is a basic and important relation between Toeplitz and Hankel operators:

$$
T_{F G}-T_{F} T_{G}=H_{F^{*}}^{*} H_{G} .
$$

The normality, subnormality and hyponormality of a scalar Toeplitz operator have been studied in [Brown and Halmos 1963; Abrahamse 1976; Itô and Wong 1972; Cowen 1988a; 1988b; Hwang and Lee 2002]. Cowen [1988a] gave an elegant characterization of a scalar hyponormal Toeplitz operator: $T_{\varphi}$ is hyponormal if and only if there exists a $k \in H^{\infty}$ with $\|k\|_{\infty} \leq 1$ satisfying $\varphi-k \bar{\varphi} \in H^{2}$.

MSC2000: 47B35, 47B20.

Keywords: Toeplitz operator, Hankel operator, hyponormal operator, inner matrix.

Research by Gu was partially supported by National Science Foundation Grant DMS-0075127. The research of all authors was partially supported by National Science Foundation Grant DMS-0097329. 
Here we discuss the hyponormality of a block Toeplitz operator. An extension of Cowen's result to the generalized Toeplitz operators was obtained in [Gu 1994]. Since the symbol of a generalized Toeplitz operator discussed in that reference can be operator-valued, it is assumed to be normal. Because the symbol of a block Toeplitz operator is a matrix-valued function, here we obtain more refined and explicit results than in the general operator-valued case. In particular we show the hyponormality of $T_{G}$ will force $G$ to be normal, that is, $G^{*} G=G G^{*}$. We prove that $T_{G}$ is hyponormal if and only if $G^{*} G=G G^{*}$ and there exists a matrix $K(z) \in$ $H^{\infty}\left(\mathbb{C}^{n \times n}\right)$ such that $\|K\|_{\infty} \leq 1$ and $G-K G^{*} \in H^{\infty}\left(\mathbb{C}^{n \times n}\right)$. The extra condition in the block case, $G^{*} G=G G^{*}$, indicates, for example, that the hyponormality of a block Toeplitz operator $T_{G}$ does depend on the constant term $G(0)$, while the hyponormality of a scalar Toeplitz operator $T_{\varphi}$ does not depend on $\varphi(0)$.

Efforts have been made to give more explicit conditions for the hyponormality of a scalar Toeplitz operator $T_{\varphi}$. That is, how can we actually check if there is such a $k \in H^{\infty}$ with $\|k\|_{\infty} \leq 1$ satisfying $\varphi-k \bar{\varphi} \in H^{2}$ ? It has been shown in [Zhu 1995; Gu 1994; Gu and Shapiro 2001] that verifying this condition is equivalent to a certain interpolation problem. It is more difficult to find a matrix $K(z) \in$ $H^{\infty}\left(\mathbb{C}^{n \times n}\right)$ such that $\|K\|_{\infty} \leq 1$ and $G-K G^{*} \in H^{\infty}\left(\mathbb{C}^{n \times n}\right)$ for the matrix-valued function $G$. One obvious reason is that matrix multiplication is not commutative. Another lies in the difficulty of factoring or dividing matrix-valued functions. We will show that, as in the scalar case, if $G(z)$ is a trigonometric matrix polynomial with invertible leading coefficient, then finding such a $K$ is equivalent to solving a matrix-valued Carathéodory interpolation problem. We note that detailed analysis of the hyponormality of $T_{\varphi}$ with scalar trigonometric or rational symbol $\varphi$ was done recently in [Farenick and Lee 1996; Hwang and Lee 2002; Gu and Shapiro 2001].

More explicit conditions are given for the hyponormality of $T_{G}$ with $G$ satisfying an extremal condition. As a pleasant surprise, we obtain a simple characterization of a class of normal block Toeplitz operators.

As alluded to above, Hankel operators play an essential role in our approach. Let $G \in L^{\infty}\left(\mathbb{C}^{n \times n}\right)$. Since the kernel of a block Hankel operator is an invariant subspace, by the Beurling-Lax-Halmos Theorem, Ker $H_{G}=\Omega(z) H^{2}\left(\mathbb{C}^{m}\right)$ for some inner matrix $\Omega(z)$ and $m \leq n$. In the next section we explore the connection between the symbol $G$ and the inner function $\Omega(z)$. In particular we answer the question for what $G$, will $\Omega(z)$ be a square inner function (i.e., $m=n$ )? For a scalar analytic polynomial $\varphi$, it is easy to see that $\operatorname{Ker} H_{\bar{\varphi}}=z^{k} H^{2}(\mathbb{C})$ where $k$ is the degree of $\varphi$. But for a matrix analytic polynomial $F$, it is not trivial to identify Ker $H_{F^{*}}$. Those discussions appear important for the study of the hyponormality of a single block Toeplitz operator in this paper and for the joint hyponormality of several block Toeplitz opeartors to be discussed in a future article. The results about kernels of block Hankel opearators are of independent interest. 


\section{The Kernel of a block Hankel operator}

An inner matrix $\Omega(z) \in H^{2}\left(\mathbb{C}^{n \times m}\right)$ is one satisfying $\Omega(z)^{*} \Omega(z)=I_{m}$ for all $z$ on the unit circle. The kernel of a Hankel operator $H_{F}$ is an invariant subspace. By the Beurling-Lax-Halmos Theorem,

$$
\operatorname{Ker} H_{F}=\Omega(z) H^{2}\left(\mathbb{C}^{m}\right)
$$

for some inner matrix $\Omega(z)$. A question relevant to our work is how the symbol $F$ is related to the inner matrix $\Omega(z)$. In particular, for which symbol $F$ is $\Omega(z)$ a square inner matrix?

Recall a scalar function $f \in L^{2}$ is of bounded type if $f$ is a quotient $p / q$ for some $p, q \in H^{\infty}$.

Definition 2.1. Let $F=\left[f_{i j}\right] \in L^{\infty}\left(\mathbb{C}^{n \times n}\right)$. We say the matrix-valued function $F$ is of bounded type if each entry $f_{i j}$ is of bounded type.

An equivalent definition is that $F$ is of bounded type if $F(z)=P(z) Q(z)^{-1}$ for some $P(z), Q(z) \in H^{\infty}\left(\mathbb{C}^{n \times n}\right)$ with $\operatorname{det} Q(z)$ not identically zero.

Theorem 2.2. Let $F=\left[f_{i j}\right] \in L^{\infty}\left(\mathbb{C}^{n \times n}\right)$. $\operatorname{Ker} H_{F}=\Theta(z) H^{2}\left(\mathbb{C}^{n}\right)$ for some square inner function $\Theta(z)$ if and only if $F$ is of bounded type.

Proof. Let $\left\{e_{1}, e_{2}, \ldots, e_{n}\right\}$ be the standard basis of $\mathbb{C}^{n}$. If $\operatorname{Ker} H_{F}=\Theta(z) H^{2}\left(\mathbb{C}^{n}\right)$ for some square inner function $\Theta(z)$, then

$$
(I-P)\left(F \Theta\left[\begin{array}{llll}
e_{1} & e_{2} & \cdots & e_{n}
\end{array}\right]\right)=0 .
$$

Therefore

$$
F(z) \Theta(z)=A(z) \text { for some } A(z) \in H^{\infty}\left(\mathbb{C}^{n \times n}\right) .
$$

Multiply both sides by adj $\Theta(z)$ gives

$$
F(z) \operatorname{det} \Theta(z)=A(z) \operatorname{adj} \Theta(z) .
$$

Since $\operatorname{adj} \Theta(z) \in H^{\infty}\left(\mathbb{C}^{n \times n}\right)$ and $\operatorname{det} \Theta(z)$ is a scalar inner function, $F(z)$ is of bounded type.

Now assume $f_{i j}$ is of bounded type. Write $f_{i j}=p_{i j} / \omega_{i j}=p_{i j} \bar{\omega}_{i j}$ where $p_{i j}$, $\omega_{i j} \in H^{\infty}$ and $\omega_{i j}$ are inner. Thus

$$
F(z)=\left[f_{i j}\right]=\left[p_{i j} \bar{\omega}_{i j}\right]=\overline{\theta(z)} B(z),
$$

where $\theta=\prod_{i, j=1}^{n} \omega_{i j}$ is a scalar inner function and $B(z) \in H^{\infty}\left(\mathbb{C}^{n \times n}\right)$. It is clear that

$$
\operatorname{Ker} H_{F} \supset \theta I_{n} H^{2}\left(\mathbb{C}^{n}\right) .
$$

If $\operatorname{Ker} H_{F}=\Omega(z) H^{2}\left(\mathbb{C}^{m}\right)$ for some inner matrix $\Omega(z)$, then

$$
\Omega(z) H^{2}\left(\mathbb{C}^{m}\right) \supset \theta I_{n} H^{2}\left(\mathbb{C}^{n}\right) .
$$


Therefore

$$
\theta I_{n}=\left[\begin{array}{llll}
\theta e_{1} & \theta e_{2} & \cdots & \theta e_{n}
\end{array}\right]=\Omega(z) Q(z)
$$

for some $Q(z) \in H^{\infty}\left(\mathbb{C}^{m \times n}\right)$. Since $m \leq n$, this can happen only when $m=n$. The proof is complete.

As a consequence of the proof we obtain a characterization of matrix-valued functions of bounded type:

Corollary 2.3. Let $F=\left[f_{i j}\right] \in L^{2}\left(\mathbb{C}^{n \times n}\right)$. Then $F$ is of bounded type if and only if $F(z)=\overline{\theta(z)} A(z)$ where $A(z) \in H^{2}\left(\mathbb{C}^{n \times n}\right)$ and $\theta(z)$ is a scalar inner function.

Definition 2.4. Let $A(z), B(z) \in H^{2}\left(\mathbb{C}^{n \times n}\right)$. We say $A(z)$ and $B(z)$ are not right coprime if there exists a nonconstant inner matrix $\Delta(z)$ such that

$$
A(z)=A_{1}(z) \Delta(z), B(z)=B_{1}(z) \Delta(z)
$$

for some $A_{1}(z), B_{1}(z) \in H^{2}\left(\mathbb{C}^{n \times n}\right)$.

Corollary 2.5. Let $F=\left[f_{i j}\right] \in L^{\infty}\left(\mathbb{C}^{n \times n}\right)$. $\operatorname{Ker} H_{F}=\Theta(z) H^{2}\left(\mathbb{C}^{n}\right)$ for some square inner matrix $\Theta(z)$ if and only if $F(z)=A(z) \Theta^{*}(z)$ where $A(z) \in H^{\infty}\left(\mathbb{C}^{n \times n}\right)$ and $A(z)$ and $\Theta(z)$ are right coprime.

Proof. We first prove the sufficiency. Assume $F(z)=A(z) \Theta^{*}(z)$, where $A(z) \in$ $H^{\infty}\left(\mathbb{C}^{n \times n}\right)$ and $A(z)$ and $\Theta(z)$ are right coprime. It is clear that $F(z)$ is of bounded type and $\operatorname{Ker} H_{F} \supset \Theta(z) H^{2}\left(\mathbb{C}^{n}\right)$. If $\operatorname{Ker} H_{F}=\Omega(z) H^{2}\left(\mathbb{C}^{n}\right)$ for some square inner matrix $\Omega(z)$, as in the proof of the theorem above, we also have

$$
F(z)=A_{1}(z) \Omega^{*}(z) \text { for some } A_{1}(z) \in H^{\infty}\left(\mathbb{C}^{n \times n}\right) .
$$

We need to show that $\Omega(z)=\Theta(z)$ (up to a right unitary constant). Since

$$
\operatorname{Ker} H_{F}=\Omega(z) H^{2}\left(\mathbb{C}^{n}\right) \supset \Theta(z) H^{2}\left(\mathbb{C}^{n}\right),
$$

there exists an inner matrix $\Delta(z)$ such that

$$
\Theta(z)=\Omega(z) \Delta(z)
$$

Thus

$$
F(z)=A_{1}(z) \Omega^{*}(z)=A(z) \Theta^{*}(z)=A(z) \Delta^{*}(z) \Omega^{*}(z) .
$$

Equivalently,

$$
A(z)=A_{1}(z) \Delta(z),
$$

that is, $\Delta(z)$ is a common inner (right) factor of $A(z)$ and $\Theta(z)$. Since $A(z)$ and $\Theta(z)$ are right coprime, $\Delta(z)$ is a unitary constant matrix.

The proof of necessity is similar.

In the scalar case, if $\varphi\left(e^{i \theta}\right)=\sum_{m=-k}^{-1} \varphi_{m} e^{i m \theta}$ for some $k \geq 1$ and $\varphi_{-k} \neq 0$, then $\operatorname{Ker} H_{\varphi}=z^{k} H^{2}$. For a matrix polynomial $F$, it is not as trivial to identify $\operatorname{Ker} H_{F}$. We will deal with the following case frequently in this paper. 
Lemma 2.6. Assume $F \in L^{\infty}\left(\mathbb{C}^{n \times n}\right)$ can be written as $F\left(e^{i \theta}\right)=\sum_{m=-k}^{-1} F_{m} e^{i m \theta}$.

(a) Ker $H_{F}=z^{k} H^{2}\left(\mathbb{C}^{n}\right)$ if and only if $F_{-k}$ is invertible. In this case rank $H_{F}=n k$.

(b) Assume that $F_{-k}$ is invertible and that $G \in L^{\infty}\left(\mathbb{C}^{n \times n}\right)$ can be written as $G\left(e^{i \theta}\right)=\sum_{m=-l}^{-1} G_{m} e^{i m \theta}$ with $l \leq k$. Then $\operatorname{Ker}\left(H_{F}^{*} H_{G}\right)=\operatorname{Ker} H_{G}$ and $\operatorname{rank}\left(H_{F}^{*} H_{G}\right)=\operatorname{rank} H_{G}$.

Proof. Assume $F_{-k}$ is not invertible. Let $v \in \mathbb{C}^{n}$ be such that $F_{-k} v=0$. Note that

$$
(I-P)\left(F v z^{k-1}\right)=F_{-k} v \bar{z}=0 .
$$

That is, $v z^{k-1} \in \operatorname{Ker} H_{F}$. Therefore $\operatorname{Ker} H_{F} \neq z^{k} H^{2}\left(\mathbb{C}^{n}\right)$. If $F_{-k}$ is invertible, it is easy to verify that $\operatorname{Ker} H_{F}=z^{k} H^{2}\left(\mathbb{C}^{n}\right)$. Since the codimension of $z^{k} H^{2}\left(\mathbb{C}^{n}\right)$ is $n k$, the rank of $H_{F}$ is $n k$.

Now we prove (b). Since $H_{G}^{*}=H_{\widetilde{G}}$ and $\widetilde{G}$ is conjugate analytic of degree $l$, Ker $H_{G}^{*} \supset z^{l} H^{2}\left(\mathbb{C}^{n}\right)$. Therefore

$$
\text { Range } H_{G} \subset \operatorname{Ker}\left(H_{G}^{*}\right)^{\perp} \subset H^{2}\left(\mathbb{C}^{n}\right) \ominus z^{l} H^{2}\left(\mathbb{C}^{n}\right) .
$$

If $h \in \operatorname{Ker}\left(H_{F}^{*} H_{G}\right)$ or $H_{F}^{*} H_{G} h=0$, then $H_{G} h \in \operatorname{Ker} H_{F}^{*}$. By part (a), Ker $H_{F}^{*}=$ $\operatorname{Ker} H_{\widetilde{F}}=z^{k} H^{2}\left(\mathbb{C}^{n}\right)$. So $H_{G} h \in \operatorname{Range}\left(H_{G}\right) \cap z^{k} H^{2}\left(\mathbb{C}^{n}\right)$. By the assumption $l \leq k$, we have Range $\left(H_{G}\right) \cap z^{k} H^{2}\left(\mathbb{C}^{n}\right)=\{0\}$. We conclude $H_{G} h=0$. This proves $\operatorname{Ker}\left(H_{F}^{*} H_{G}\right)=\operatorname{Ker} H_{G}$. The proof is complete.

In the case where $F_{-k}$ may be singular, we have the following result for $\operatorname{Ker} H_{F}$ :

Lemma 2.7. Assume $F \in L^{\infty}\left(\mathbb{C}^{n \times n}\right)$ can be written as $F\left(e^{i \theta}\right)=\sum_{m=-k}^{-1} F_{m} e^{i m \theta}$ with $F_{-k} \neq 0$. Then

$$
\operatorname{Ker} H_{F} \supset z^{k} H^{2}\left(\mathbb{C}^{n}\right) \quad \text { and } \quad \operatorname{Ker} H_{F} \nsupseteq z^{k-1} H^{2}\left(\mathbb{C}^{n}\right) \text {. }
$$

Set $\operatorname{Ker} H_{F}=\Theta(z) H^{2}\left(\mathbb{C}^{n}\right)$ for some square inner function $\Theta(z)$. Then there exists an inner function $\Omega(z)$ such that

$$
\Theta(z) \Omega(z)=\Omega(z) \Theta(z)=z^{k} I_{n} \quad \text { and } \quad \Omega(0) \neq 0 .
$$

Proof. Relation (2-1) follows from the definitions. Since

$$
\operatorname{Ker} H_{F}=\Theta(z) H^{2}\left(\mathbb{C}^{n}\right) \supset z^{k} H^{2}\left(\mathbb{C}^{n}\right),
$$

there exists $\Omega(z) \in H^{\infty}\left(\mathbb{C}^{n \times n}\right)$ such that $\Theta(z) \Omega(z)=z^{k} I_{n}$. That is, $\Omega(z)=z^{k} \Theta^{*}(z)$ on the unit circle. Thus $\Omega(z)$ is in fact an inner function. We prove $\Omega(0) \neq 0$ by contradiction. Assume $\Omega(z)=z^{l} \Omega_{1}(z)$ for some inner function $\Omega_{1}(z)$ and $l \geq 1$. Then

$$
\Theta(z) \Omega_{1}(z)=z^{k-l} I_{n} \quad \text { and } \quad \Theta^{*}(z)=\bar{z}^{k-l} \Omega_{1}(z) .
$$

Since Ker $H_{F}=\Theta(z) H^{2}\left(\mathbb{C}^{n}\right)$, by Corollary 2.5, $F(z)=A(z) \Theta^{*}(z)$. Therefore $F(z)=A(z) \Omega_{1}(z) \bar{z}^{k-l}$. But this implies that Ker $H_{F} \supset z^{k-1} H^{2}\left(\mathbb{C}^{n}\right)$, contradicting 
(2-1). The relation $\Omega(z) \Theta(z)=z^{k} I_{n}$ is valid for each fixed nonzero $z$ inside the unit disk because $\Omega(z)$ is the inverse of $\Theta(z)$, and by continuity, $\Omega(z) \Theta(z)=z^{k} I_{n}$. The proof is complete.

Here is an example of $\operatorname{Ker} H_{F}$, where $F_{-k}$ is singular:

Example 2.8. Set

$$
\begin{gathered}
F=\left[\begin{array}{rr}
\bar{z}^{2}+\bar{z} & \bar{z}^{2} \\
\bar{z}^{2} & \bar{z}^{2}+\bar{z}
\end{array}\right]=\left[\begin{array}{ll}
1 & 1 \\
1 & 1
\end{array}\right] \bar{z}^{2}+\left[\begin{array}{ll}
1 & 0 \\
0 & 1
\end{array}\right] \bar{z} \\
\Theta(z)=\frac{1}{\sqrt{2}} z\left[\begin{array}{rr}
1 & z \\
-1 & z
\end{array}\right], \quad \Omega(z)=z^{2} \Theta^{*}(z)=\frac{1}{\sqrt{2}}\left[\begin{array}{rr}
z & -z \\
1 & 1
\end{array}\right] .
\end{gathered}
$$

Then $\operatorname{Ker} H_{F}=\Theta(z) H^{2}\left(\mathbb{C}^{2}\right)$ and $\Theta(z) \Omega(z)=z^{2} I_{2}$.

We end this section with an example of $\operatorname{Ker} H_{F}=\Omega(z) H^{2}\left(\mathbb{C}^{m}\right)$ when $\Omega(z)$ is not a square inner matrix.

Example 2.9. Let $\theta_{0}, \theta_{1}$ and $\theta_{2}$ be three scalar inner functions such that $\theta_{1}$ and $\theta_{2}$ are coprime. Let $q \in L^{\infty}$ be such that $\operatorname{Ker} H_{q}=\{0\}$. Set

$$
\Omega(z)=\frac{1}{\sqrt{2}}\left[\begin{array}{c}
\theta_{0} \theta_{1} \\
\theta_{0} \theta_{2}
\end{array}\right], \quad F=\left[\begin{array}{cc}
\overline{\theta_{0} \theta_{1}} & \overline{\theta_{0} \theta_{2}} \\
q \theta_{2} & -q \theta_{1}
\end{array}\right] .
$$

Then $\operatorname{Ker} H_{F}=\Omega(z) H^{2}$. For $k=\left[k_{1}, k_{2}\right]^{T} \in H^{2}\left(\mathbb{C}^{2}\right)$, the equality $H_{F} k=0$ is equivalent to

$$
\overline{\theta_{0} \theta_{1}} k_{1}+\overline{\theta_{0} \theta_{2}} k_{2}=p_{1} \quad \text { and } \quad q \theta_{2} k_{1}-q \theta_{1} k_{2}=p_{2} \quad \text { for some } p_{1}, p_{2} \in H^{2} .
$$

Since $\operatorname{Ker} H_{q}=\{0\}$, we have $\theta_{2} k_{1}=\theta_{1} k_{2}$. Since $\theta_{1}$ and $\theta_{2}$ are coprime, $k_{1}=\theta_{1} h_{1}$ and $k_{2}=\theta_{2} h_{1}$ for some $h_{1} \in H^{2}$. Now the first equation above becomes $2 \overline{\theta_{0}} h_{1}=p_{1}$. Therefore

$$
k=\left[k_{1}, k_{2}\right]^{T}=\frac{1}{2}\left[\theta_{0} \theta_{1} p_{1}, \theta_{0} \theta_{2} p_{1}\right]^{T}=\Omega(z) \sqrt{2} p_{1} \in \Omega(z) H^{2} .
$$

\section{Hyponormality of a block Toeplitz operator}

C. C. Cowen [1988a] characterized the hyponormality of a single Toeplitz operator in terms of its symbol. For a given $\varphi \in L^{2}$ we may write $\varphi=\varphi_{+}+\bar{\varphi}_{-}$with $\varphi_{+}, \varphi_{-} \in H^{2}$. Cowen's theorem states that

$T_{\varphi}$ is hyponormal if and only if there exists a $k \in H^{\infty}$ with $\|k\|_{\infty} \leq 1$ satisfying $\bar{\varphi}_{-}-k \bar{\varphi}_{+} \in H^{2}$.

An equivalent condition in [Nakazi and Takahashi 1993] is that $\varphi-k \bar{\varphi} \in H^{\infty}$. A generalization of Cowen's result for the hyponormality of generalized Toeplitz operators was obtained in [Gu 1994]. Since the symbol of a generalized Toeplitz operator discussed in that reference can be operator-valued, it is assumed to be 
normal. Here our contribution is to note that for a block Toeplitz operator $T_{G}$, the hyponormality of $T_{G}$ will force $G$ to be normal. That is, $G^{*} G=G G^{*}$. This follows essentially from the fact that a hyponormal constant matrix is normal.

Let $H^{2}(\mathbb{D})$ be the scalar Hardy space of the unit disk $\mathbb{D}$ and let $\partial \mathbb{D}$ be the unit circle. Let $k_{z}(w)$ be the normalized reproducing kernel of $H^{2}$,

$$
k_{z}(w)=\frac{\left(1-|z|^{2}\right)^{1 / 2}}{1-\bar{z} w} .
$$

For $f \in L^{2}(\partial \mathbb{D})$ with Fourier series

$$
f\left(e^{i \theta}\right)=\sum_{m=-\infty}^{\infty} f_{m} e^{i m \theta},
$$

the Poisson integral of $f$, still denoted by $f$, is

$$
f(z)=\left\langle f(w) k_{z}(w), k_{z}(w)\right\rangle=\sum_{m=-\infty}^{-1} f_{m} \bar{z}^{|m|}+\sum_{m=0}^{\infty} f_{m} z^{m}, \quad z \in \mathbb{D} .
$$

The following lemma is probably known. We include a short proof.

Lemma 3.1. Let $F \in L^{\infty}\left(\mathbb{C}^{n \times n}\right)$. If $T_{F}$ is a positive operator on $H^{2}\left(\mathbb{C}^{n}\right)$, then $F(z)$ is a positive semidefinite matrix for all $z \in \mathbb{D}$.

Proof. Without loss of generality, we prove the lemma for $n=2$. Let $h(w)=$ $\left[\alpha_{1} k_{z}(w), \alpha_{2} k_{z}(w)\right]^{T}$ be a column vector in $H^{2}\left(\mathbb{C}^{2}\right)$, where $\alpha_{1}$ and $\alpha_{2}$ are complex numbers. Set $F(w)=\left[f_{i j}\right]$. The lemma follows from the following computation:

$\left\langle T_{F} h, h\right\rangle=\langle F h, h\rangle=\sum_{i, j=1}^{2}\left\langle f_{i j}(w) k_{z}(w), k_{z}(w)\right\rangle \alpha_{j} \bar{\alpha}_{i}=\sum_{i, j=1}^{2} f_{i j}(z) \alpha_{j} \bar{\alpha}_{i} \geq 0, \quad z \in D$.

We will often make use of the following identities.

Lemma 3.2. For $G, \Theta \in H^{\infty}\left(\mathbb{C}^{n \times n}\right)$ with $\Theta$ inner and $F \in L^{\infty}\left(\mathbb{C}^{n \times n}\right)$, $H_{F} T_{G}=H_{F G}, \quad H_{G F}=T_{\widetilde{G}}^{*} H_{F}, \quad$ and $\quad H_{F}^{*} H_{F}-H_{\Theta F}^{*} H_{\Theta F}=H_{F}^{*} H_{\Theta^{*}} H_{\Theta^{*}}^{*} H_{F}$.

Proof. The identity $H_{F} T_{G}=H_{F G}$ follows from the analyticity of $G$. The identity $H_{G F}=T_{\widetilde{G}}^{*} H_{F}$ can be obtained essentially by taking the adjoint of $H_{F} T_{G}=H_{F G}$. Note that

$$
\begin{aligned}
H_{F}^{*} H_{F}-H_{\Theta F}^{*} H_{\Theta F} & =H_{F}^{*} H_{F}-H_{F}^{*} T_{\widetilde{\Theta}} T_{\widetilde{\Theta}}^{*} H_{F} \\
& =H_{F}^{*}\left(I-T_{\widetilde{\Theta}} T_{\widetilde{\Theta}}^{*}\right) H_{F}=H_{F}^{*} H_{\widetilde{\Theta}^{*}}^{*} H_{\widetilde{\Theta}^{*}} H_{F} \\
& =H_{F}^{*} H_{\Theta^{*}} H_{\Theta^{*}}^{*} H_{F} .
\end{aligned}
$$


Theorem 3.3. Let $G \in L^{\infty}\left(\mathbb{C}^{n \times n}\right)$. The block Toeplitz operator $T_{G}$ is hyponormal if and only if the following two conditions hold:

(1) $G$ is normal, i.e. $G^{*}(z) G(z)=G(z) G^{*}(z)$ for almost every $z \in \partial \mathbb{D}$.

(2) There exists a matrix $K(z) \in H^{\infty}\left(\mathbb{C}^{n \times n}\right)$ such that $\|K\|_{\infty} \leq 1$ and $G-K G^{*} \in$ $H^{\infty}\left(\mathbb{C}^{n \times n}\right)$.

Proof. Assuming $T_{G}$ is hyponormal, we have

$$
\begin{aligned}
T_{G}^{*} T_{G}-T_{G} T_{G}^{*} & =T_{G}^{*} T_{G}-T_{G^{*} G}+T_{G G^{*}}-T_{G} T_{G}^{*}+T_{G^{*} G-G G^{*}} \\
& =H_{G^{*}}^{*} H_{G^{*}}-H_{G}^{*} H_{G}+T_{G^{*} G-G G^{*}} \geq 0 .
\end{aligned}
$$

Therefore, for all $m \geq 1, h \in H^{2}\left(\mathbb{C}^{n}\right)$,

$$
\left\langle H_{G^{*}}^{*} H_{G^{*}} z^{m} h, z^{m} h\right\rangle-\left\langle H_{G}^{*} H_{G} z^{m} h, z^{m} h\right\rangle+\left\langle T_{G^{*} G-G G^{*}} z^{m} h, z^{m} h\right\rangle \geq 0 .
$$

Note that

$$
\begin{aligned}
\lim _{m \rightarrow \infty} H_{G^{*}}\left(z^{m} h\right) & =(I-P)\left(z^{m} G^{*} h\right)=0, \\
\left\langle T_{G^{*} G-G G^{*}} z^{m} h, z^{m} h\right\rangle & =\left\langle S^{* m} T_{G^{*} G-G G^{*}} S^{m} h, h\right\rangle=\left\langle T_{G^{*} G-G G^{*}} h, h\right\rangle .
\end{aligned}
$$

Taking the limit in (3-1), we have

$$
\left\langle T_{G^{*} G-G G^{*}} h, h\right\rangle \geq 0 .
$$

By Lemma 3.1, the Poisson integral of $G^{*} G-G G^{*}$ is positive semidefinite for $z \in \mathbb{D}$. The Poisson integral of $G^{*} G-G G^{*}$ is in general not equal to $G^{*}(z) G(z)-$ $G(z) G^{*}(z)$ for $z \in \mathbb{D}$. By taking nontangential limits, we do know that the limit of the Poisson integral of $G^{*} G-G G^{*}$ is the same as $G^{*}(z) G(z)-G(z) G^{*}(z)$ for almost every $z \in \partial \mathbb{D}$. Therefore $G^{*}(z) G(z)-G(z) G^{*}(z) \geq 0$. But $G(z)$ is a finite matrix, so $G^{*} G=G G^{*}$ on $\partial \mathbb{D}$.

Now equation (3-1) becomes

$$
H_{G^{*}}^{*} H_{G^{*}} \geq H_{G}^{*} H_{G}
$$

By [Gu 1994, Corollary 2] there exists a contractive co-analytic Toeplitz operator $T_{\widetilde{K}}^{*}$ with $K(z) \in H^{\infty}\left(\mathbb{C}^{n \times n}\right)$ and $H_{G}=T_{\widetilde{K}}^{*} H_{G^{*}}$. Thus $\|K(z)\|_{\infty}=\left\|T_{\widetilde{K}}^{*}\right\| \leq 1$. By Lemma 3.2, $T_{\widetilde{K}}^{*} H_{G^{*}}=H_{K G^{*}}$, so $H_{G}=T_{\widetilde{K}}^{*} H_{G^{*}}=H_{K G^{*}}$ and $G-K G^{*} \in H^{\infty}\left(\mathbb{C}^{n \times n}\right)$. Sufficiency follows essentially from the preceding argument.

Condition (2) in Theorem 3.3 is analogous to the condition in Cowen's theorem for a scalar hyponormal Toeplitz operator. But condition (1) is new for the block case. This implies, for example, that the hyponormality of a block Toeplitz operator $T_{G}$ does depend on the constant term $G(0)$, while the hyponormality of a scalar Toeplitz operator $T_{\varphi}$ does not depend on $\varphi(0)$. If $\varphi$ is analytic, then the scalar Toeplitz operator $T_{\varphi}$ is hyponormal. In the block case we have the following results. 
Corollary 3.4. If $G \in H^{\infty}\left(\mathbb{C}^{n \times n}\right)$, then the analytic block Toeplitz operator $T_{G}$ is hyponormal if and only if $G^{*}(z) G(z)=G(z) G^{*}(z)$ for almost every $z \in \partial \mathbb{D}$.

Corollary 3.5. Let $G \in L^{\infty}\left(\mathbb{C}^{n \times n}\right)$, and assume $T_{G}$ is hyponormal.

(1) $\operatorname{Ker} H_{G} \supset \operatorname{Ker} H_{G^{*}}$.

(2) If $G^{*}$ is of bounded type, so is $G$.

Proof. Statement (1) follows from the proof of Theorem 3.3. If $T_{G}$ is hyponormal, there exists a matrix such that $\|K\|_{\infty} \leq 1$ and

$$
G-K G^{*}=C(z) \text { for some } C(z) \in H^{\infty}\left(\mathbb{C}^{n \times n}\right) .
$$

If $G^{*}$ is of bounded type, by Corollary $2.3, G^{*}=\overline{\theta(z)} A(z)$ where $\theta(z)$ is scalar inner function and $A(z) \in H^{\infty}\left(\mathbb{C}^{n \times n}\right)$, thus

$$
G=K G^{*}+C(z)=\overline{\theta(z)}(K A(z)+\theta(z) C(z)) .
$$

That is, $G$ is of bounded type.

Remark 3.6. In the scalar case, Abrahamse [1976] noted that if $\varphi$ is not analytic and $T_{\varphi}$ is hyponormal, then $\varphi$ is of bounded type if and only if $\bar{\varphi}$ is of bounded type, which can also be seen from the above argument. The following example shows $T_{G}$ is hyponormal, $G$ is not analytic and is of bounded type, but $G^{*}$ is not of bounded type. Let $f \in H^{\infty}$ be such that $\bar{f}$ is not of bounded type and set

$$
G=\left[\begin{array}{cc}
z+\bar{z} & 0 \\
0 & f
\end{array}\right], \quad G^{*}=\left[\begin{array}{cc}
z+\bar{z} & 0 \\
0 & \bar{f}
\end{array}\right]
$$

It is clear that $G^{*}$ is not of bounded type. Since $G$ is diagonal, $G^{*}(z) G(z)=$ $G(z) G^{*}(z)$. Furthermore

$$
G-\left[\begin{array}{ll}
1 & 0 \\
0 & 0
\end{array}\right] G^{*}=\left[\begin{array}{ll}
0 & 0 \\
0 & f
\end{array}\right] .
$$

By Theorem 3.3, $T_{G}$ is hyponormal.

Recent efforts have been made to give more explicit conditions for the hyponormality of the scalar Toeplitz operator $T_{\varphi}$. That is, how can we actually check if there is such a $k \in H^{\infty}$ with $\|k\|_{\infty} \leq 1$ satisfying $\bar{\varphi}_{-}-k \bar{\varphi}_{+} \in H^{2}$ ? Zhu [1995] showed that verifying this condition for a trigonometric polynomial symbol $\varphi$ is a Carathéodory interpolation problem. Gu [1994] showed that verifying this condition for a rational symbol $\varphi$ is a tangential Hermite-Fejér interpolation problem. Gu and Shapiro [2001] showed that verifying this condition for a bounded type symbol $\varphi$ is a Sarason [1967] interpolation problem. We remark that if $T_{\varphi}$ is hyponormal and its symbol $\varphi$ is not of bounded type, then there exists only one 
$k \in H^{\infty}$ satisfying $\bar{\varphi}_{-}-k \bar{\varphi}_{+} \in H^{2}$. Because if $k_{1}, k_{2} \in H^{\infty}$ satisfy $\varphi-k_{1} \bar{\varphi}=p_{1}$ and $\varphi-k_{2} \bar{\varphi}=p_{2}$ for some $p_{1}, p_{2} \in H^{\infty}$, then

$$
k_{1} \bar{\varphi}-k_{2} \bar{\varphi}=p_{2}-p_{1} \quad \text { and } \quad \bar{\varphi}=\left(p_{2}-p_{1}\right) /\left(k_{2}-k_{1}\right) .
$$

So $\bar{\varphi}$ is of bounded type and hence $\varphi$ is of bounded type, a contradiction. We note that a detailed analysis of the hyponormality of $T_{\varphi}$ with trigonometric polynomial symbol $\varphi$ was done by Lee and his collaborators Farenick and Lee 1996; Hwang et al. 1999; Hwang and Lee 2002.

It is more difficult to verify condition (2) in Theorem 3.3 for $G \in L^{\infty}\left(\mathbb{C}^{n \times n}\right)$. One obvious reason is that matrix multiplication is not commutative. Another reason lies in the difficult of factoring or dividing matrix-valued functions. We will show that, as in the scalar case, if $G(z)$ is a trigonometric matrix polynomial with invertible leading coefficient, verifying condition (2) for $G$ amounts to a matrix Carathéodory interpolation problem. This will be done in Section 5. In the next section we try to understand condition (2) for $G$ satisfying an extremal condition.

\section{Hyponormality of $T_{G}$ with $\left\|G_{+}\right\|_{2}=\left\|G_{-}\right\|_{2}$}

For a matrix-valued function $M \in L^{2}\left(\mathbb{C}^{n \times n}\right)$, let

$$
M\left(e^{i \theta}\right)=\left[m_{k l}\left(e^{i \theta}\right)\right]_{n \times n}=\sum_{k=-\infty}^{\infty} M_{k} e^{i k \theta}
$$

be the Fourier series of $M\left(e^{i \theta}\right)$. The 2-norm of $M$ is defined by

$$
\|M\|_{2}^{2}:=\frac{1}{2 \pi} \int_{0}^{2 \pi} \operatorname{tr}\left(M\left(e^{i \theta}\right)^{*} M\left(e^{i \theta}\right)\right) d \theta=\frac{1}{2 \pi} \sum_{n=-\infty}^{\infty} \operatorname{tr}\left(M_{k}^{*} M_{k}\right)
$$

where $\operatorname{tr} A$ is the trace of matrix $A$.

The condition $\left\|\varphi_{+}\right\|_{2}=\left\|\varphi_{-}\right\|_{2}$ for the scalar symbol $\varphi$ was introduced in [Gu and Shapiro 2001]. This condition was inspired by the work of Farenick and Lee [1997] on the hyponormality of $T_{\varphi}$ with a circulant trigonometric polynomial symbol $\varphi$. Here we view this condition naturally as an extremal condition. Let $G=G_{+}+$ $G_{0}+G_{-}^{*} \in L^{\infty}\left(\mathbb{C}^{n \times n}\right)$ where $G_{+}, G_{-} \in H^{2}\left(\mathbb{C}^{n \times n}\right)$ and $G_{0}$ is a constant matrix. By Theorem 3.3, if $T_{G}$ is hyponormal then there exists a matrix $K(z) \in H^{\infty}\left(\mathbb{C}^{n \times n}\right)$ such that $\|K\|_{\infty} \leq 1$ and $G-K G^{*} \in H^{\infty}\left(\mathbb{C}^{n \times n}\right)$. Therefore

$$
\begin{aligned}
G_{-}^{*} & =(I-P)\left(K G_{+}^{*}\right), \\
\left\|G_{-}\right\|_{2} & =\left\|G_{-}^{*}\right\|_{2}=\left\|(I-P)\left(K G_{+}^{*}\right)\right\|_{2} \\
& \leq\left\|K G_{+}^{*}\right\|_{2} \leq\|K\|_{\infty}\left\|G_{+}^{*}\right\|_{2} \leq\left\|G_{+}^{*}\right\|_{2}=\left\|G_{+}\right\|_{2} .
\end{aligned}
$$


We now characterize the hyponormality of $T_{G}$ in the extremal case $\left\|G_{+}\right\|_{2}=$ $\left\|G_{-}\right\|_{2}$.

Theorem 4.1. Let $G=G_{+}+G_{0}+G_{-}^{*} \in L^{\infty}\left(\mathbb{C}^{n \times n}\right)$. Assume $\left\|G_{+}\right\|_{2}=\left\|G_{-}\right\|_{2}$ and $\operatorname{det} G_{+}$is not identically zero. Then $T_{G}$ is hyponormal if and only if $G^{*} G=G G^{*}$ and $G_{+}=G_{-} K$ for some inner matrix $K \in H^{\infty}\left(\mathbb{C}^{n \times n}\right)$.

Proof. We prove necessity. As above, by Theorem 3.3, if $T_{G}$ is hyponormal, then there exists a matrix $K(z) \in H^{\infty}\left(\mathbb{C}^{n \times n}\right)$ such that $\|K\|_{\infty} \leq 1$ and $G_{-}^{*}-K G_{+}^{*} \in$ $H^{\infty}\left(\mathbb{C}^{n \times n}\right)$. Therefore

$$
\begin{aligned}
G_{-}^{*} & =(I-P)\left(K G_{+}^{*}\right), \\
\left\|G_{-}\right\|_{2} & =\left\|G_{-}^{*}\right\|_{2}=\left\|(I-P)\left(K G_{+}^{*}\right)\right\|_{2} \\
& \leq\left\|K G_{+}^{*}\right\|_{2} \leq\|K\|_{\infty}\left\|G_{+}^{*}\right\|_{2} \leq\left\|G_{+}^{*}\right\|_{2}=\left\|G_{+}\right\|_{2} .
\end{aligned}
$$

Since $\left\|G_{+}\right\|_{2}=\left\|G_{-}\right\|_{2}$, we must have equality everywhere. But the equality $\|(I-$ $P)\left(K G_{+}^{*}\right)\left\|_{2}=\right\| K G_{+}^{*} \|_{2}$ implies that $K G_{+}^{*} \in H^{2}\left(\mathbb{C}^{n \times n}\right)^{\perp}$. Therefore

$$
G_{-}^{*}=(I-P)\left(K G_{+}^{*}\right)=K G_{+}^{*} .
$$

Now $\left\|G_{+}^{*}\right\|_{2}=\left\|G_{-}\right\|_{2}=\left\|K G_{+}^{*}\right\|_{2}$ implies that

$$
\begin{aligned}
\left\|G_{+}^{*}\right\|_{2}^{2}-\left\|K G_{+}^{*}\right\|_{2}^{2} & =\frac{1}{2 \pi} \int_{0}^{2 \pi} \operatorname{tr}\left(G_{+} G_{+}^{*}\right)-\operatorname{tr}\left(G_{+} K^{*} K G_{+}^{*}\right) d \theta \\
& =\frac{1}{2 \pi} \int_{0}^{2 \pi} \operatorname{tr}\left(G_{+}\left(I-K^{*} K\right) G_{+}^{*}\right) d \theta=\left\|G_{+}\left(I-K^{*} K\right)^{1 / 2}\right\|_{2}^{2} .
\end{aligned}
$$

Here we use the fact that $\|K\|_{\infty} \leq 1$ and $I-K^{*} K$ is positive. Thus $G_{+}\left(I-K^{*} K\right)^{1 / 2}$ vanishes. Since $\operatorname{det} G_{+}$is analytic and $\operatorname{det} G_{+}$is nonzero almost everywhere on $\partial D$, we have $\left(I-K^{*} K\right)=0$. That is, $K$ is an inner matrix. Left multiplying (4-2) by $K^{*}$ and taking adjoints we get $G_{-} K=G_{+}$, as desired.

Sufficiency clearly follows from Theorem 3.3.

Corollary 4.2. Let $G=G_{+}+G_{-}^{*} \in L^{\infty}\left(\mathbb{C}^{n \times n}\right)$. If $G_{+}=G_{-} K=K G_{-}$, where $K$ is an inner matrix, then $T_{G}$ is hyponormal.

Proof. By the preceding theorem we only need to verify that $G^{*} G=G G^{*}$. By assumption

$$
\begin{aligned}
& G=G_{+}+G_{-}^{*}=G_{-} K+G_{-}^{*}=\left(G_{-}+G_{-}^{*} K^{*}\right) K=G^{*} K, \\
& G=G_{+}+G_{-}^{*}=K G_{-}+G_{-}^{*}=K\left(G_{-}+K^{*} G_{-}^{*}\right)=K G^{*} .
\end{aligned}
$$

Therefore

$$
G^{*} G=G^{*} K G^{*}=K G^{*} G^{*}=G G^{*} .
$$

We now identify normal block Toeplitz operators. 
Theorem 4.3. Let $G=G_{+}+G_{0}+G_{-}^{*} \in L^{\infty}\left(\mathbb{C}^{n \times n}\right)$. Assume $\operatorname{det} G_{+}$is not identically zero. Then $T_{G}$ is normal if and only if $G^{*} G=G G^{*}$ and $G_{+}=G_{-} U$ for some constant unitary matrix $U$.

Proof. $T_{G}$ is normal if and only if both $T_{G}$ and $T_{G^{*}}$ are hyponormal. If $T_{G}$ is hyponormal, then, as in (4-1), we have $\left\|G_{-}\right\|_{2} \leq\left\|G_{+}\right\|_{2}$. Similarly, if $T_{G^{*}}$ is hyponormal, $\left\|G_{+}\right\|_{2} \leq\left\|G_{-}\right\|_{2}$. Thus $\left\|G_{+}\right\|_{2}=\left\|G_{-}\right\|_{2}$. By Theorem 4.1, the hyponormality of $T_{G}$ and $T_{G^{*}}$ implies that $G_{+}=G_{-} K_{1}$ and $G_{-}=G_{+} K_{2}$ for some inner matrices $K_{1}$ and $K_{2}$. Therefore

$$
G_{+}=G_{-} K_{1}=G_{+} K_{2} K_{1} .
$$

Equivalently,

$$
G_{+}\left(I-K_{2} K_{1}\right)=0 .
$$

Since $\operatorname{det} G_{+}$is analytic and $\operatorname{det} G_{+}$is nonzero almost everywhere on $\partial D$, we have $\left(I-K_{2} K_{1}\right)=0$, and $K_{1}=K_{2}^{*}$. Therefore $K_{1}=K_{2}^{*}=U$ for some constant unitary matrix $U$.

A criterion for the normality of a block Toeplitz operator $T_{G}$ was given in [Gu and Zheng 1998, Corollary 8] for a general symbol $G$, where $\operatorname{det} G_{+}$can be identically zero. The characterization there is complicated, and it is a consequence of a result on zero products of block Hankel operators. It is a pleasant surprise that the study of the hyponormality of $T_{G}$ leads to the simple characterization above for normal block Toeplitz operators. It seems difficult to derive the condition $G_{+}=G_{-} U$ from the criteria given in [Gu and Zheng 1998, Corollary 8] under the assumption that $\operatorname{det} G_{+}$is not identically zero.

Corollary 4.4. Let $G=G_{+}+G_{-}^{*} \in L^{\infty}\left(\mathbb{C}^{n \times n}\right)$. If $G_{+}=G_{-} U=U G_{-}$for some constant unitary matrix $U$, then $T_{G}$ is normal.

The characterization of a normal scalar Toeplitz operator in [Brown and Halmos 1963] can be formulated as follows: $T_{\varphi}$ is normal if and only if $\varphi_{+}=\alpha \varphi_{-}$for some unimodular constant $\alpha$.

\section{Interpolation and block Toeplitz operators with trigonometric polynomial symbols}

We now show that verifying the hyponormality of $T_{G}$ for a class of trigonometric symbols $G$ is equivalent to a matrix Carathéodory interpolation problem. Let $G \in$ $L^{\infty}\left(\mathbb{C}^{n \times n}\right)$ be a matrix trigonometric polynomial,

$$
G=G_{+}+G_{0}+G_{-}^{*}=\sum_{j=-m}^{M} G_{j} e^{i j \theta} .
$$


We will assume that the leading coefficient $G_{M}$ is invertible and of course that $G_{-m}$ is not zero. It is more convenient to write

$$
\begin{aligned}
G_{+}^{*}=\sum_{j=1}^{M} G_{j}^{*} \bar{z}^{j}=\bar{z}^{M} A(z) & \text { with } A(z)=\sum_{j=0}^{M-1} G_{M-j}^{*} z^{j}, \\
G_{-}^{*}=\sum_{j=-m}^{-1} G_{j} \bar{z}^{j}=\bar{z}^{m} B(z) & \text { with } B(z)=\sum_{j=0}^{m-1} G_{-m+j} z^{j} .
\end{aligned}
$$

Assume $T_{G}$ is hyponormal. By Theorem 3.3, there exists $K(z) \in H^{\infty}\left(\mathbb{C}^{n \times n}\right)$ such that $\|K\|_{\infty} \leq 1$ and $G_{-}^{*}-K G_{+}^{*} \in H^{\infty}\left(\mathbb{C}^{n \times n}\right)$. That is,

$$
\bar{z}^{m} B(z)-K(z) \bar{z}^{M} A(z)=Q_{0}(z) \text { for some } Q_{0}(z) \in H^{\infty}\left(\mathbb{C}^{n \times n}\right) .
$$

If $m>M$, this becomes $B(z)-z^{m-M} K(z) A(z)=z^{m} Q_{0}(z)$. This would imply $B(0)=G_{-m}^{*}=0$. Therefore $M \geq m$ and

$$
z^{M-m} B(z)-K(z) A(z)=z^{M} Q_{0}(z) .
$$

By assumption, $A(0)=G_{M}^{*}$ is invertible. The equation above implies that

$$
K(z)=z^{M-m} \widehat{K}(z) \text {. }
$$

Equation (5-2) becomes

$$
B(z)-\widehat{K}(z) A(z)=z^{m} Q_{0}(z) .
$$

Let $\widehat{K}_{p}(z)$ be the unique analytic polynomial of degree $m-1$ satisfying (5-4). Set

$$
\widehat{K}_{p}(z)=\sum_{i=0}^{m-1} K_{i} z^{i}
$$

Using notation as in (5-1), equation (5-4) becomes

$$
\sum_{i=0}^{j} K_{i} G_{M-j+i}^{*}=G_{-m+j}, \quad j=0,1, \ldots, m-1 .
$$

Equivalently,

$$
\begin{aligned}
(5-6) & {\left[\begin{array}{llll}
K_{0} & K_{1} & \cdots & K_{m-1}
\end{array}\right]\left[\begin{array}{cccc}
G_{M}^{*} & G_{M-1}^{*} & \cdots & G_{M-m+1}^{*} \\
0 & G_{M}^{*} & \ddots & \vdots \\
\vdots & \ddots & \ddots & G_{M-1}^{*} \\
0 & \cdots & 0 & G_{M}^{*}
\end{array}\right] } \\
& \\
&
\end{aligned}
$$


The $m \times m$ block matrix on the left side is invertible since $G_{M}^{*}$ is invertible. Therefore $\widehat{K}_{p}(z)$ is uniquely determined by the equation above. Let $\widehat{K}(z) \in H^{\infty}\left(\mathbb{C}^{n \times n}\right)$ be another solution of (5-4). Then

$$
\left[\widehat{K}(z)-\widehat{K}_{p}(z)\right] A(z)=-z^{m} Q_{1}(z) \quad \text { for some } Q_{1}(z) \in H^{\infty}\left(\mathbb{C}^{n \times n}\right) .
$$

Again, by assumption, $A(0)=G_{M}^{*}$ is invertible, so the preceding equation implies

$$
\widehat{K}(z)=\widehat{K}_{p}(z)-z^{m} Q(z) \text { for some } Q(z) \in H^{\infty}\left(\mathbb{C}^{n \times n}\right) .
$$

The existence of some $K(z)=z^{M-m} \widehat{K}(z) \in H^{\infty}\left(\mathbb{C}^{n \times n}\right)$ such that $\|K\|_{\infty} \leq 1$ and $G_{-}^{*}-K G_{+}^{*} \in H^{\infty}\left(\mathbb{C}^{n \times n}\right)$ is equivalent to

$$
\inf \left\{\left\|\widehat{K}_{p}(z)-z^{m} Q(z)\right\|_{\infty}: Q(z) \in H^{\infty}\left(\mathbb{C}^{n \times n}\right)\right\} \leq 1 .
$$

This is exactly the matrix Carathéodory interpolation problem. Summarizing the discussion, we have the following result for hyponormal block Toeplitz operators with trigonometric polynomial symbols.

Theorem 5.1. Let $G$ be a matrix trigonometric polynomial with invertible leading coefficient $G_{M}$ with notation as above. The following statements are equivalent:

(a) $T_{G}$ is hyponormal.

(b) $G^{*} G=G G^{*}$ and

$$
\inf \left\{\left\|\widehat{K}_{p}(z)-z^{m} Q(z)\right\|_{\infty}: Q(z) \in H^{\infty}\left(\mathbb{C}^{n \times n}\right)\right\} \leq 1 .
$$

(c) $G^{*} G=G G^{*}$ and the $m \times m$ block Toeplitz matrix

$$
T\left(\widehat{K}_{p}\right)=\left[\begin{array}{ccc}
K_{0} & 0 & 0 \\
\vdots & \ddots & 0 \\
K_{m-1} & \cdots & K_{0}
\end{array}\right]
$$

is a contraction.

Proof. The equivalence of (a) and (b) follows from the preceding discussion. The equivalence of (b) and (c) is a classical result on Carathéodory interpolation; see [Foias and Frazho 1990].

Corollary 5.2. Let $G \in L^{\infty}\left(\mathbb{C}^{n \times n}\right)$ be a matrix trigonometric polynomial,

$$
G=G_{+}+G_{0}+G_{-}^{*}=\sum_{j=-m}^{M} G_{j} e^{i j \theta} .
$$

Assume that $G_{M}$ is invertible, $G_{-m}$ is not zero, and $T_{G}$ is hyponormal.

(a) $M \geq m$ and $G_{M} G_{M}^{*} \geq G_{-m}^{*} G_{-m}$.

(b) $n(M-m) \leq \operatorname{rank}\left[T_{G}^{*}, T_{G}\right] \leq n M$. 
(c) Let $K_{p}(z)=z^{M-m} \widehat{K}_{p}(z)$ where $\widehat{K}_{p}(z)$ is defined by (5-5) and (5-6). Then

$$
\left[T_{G}^{*}, T_{G}\right]=H_{G^{*}}^{*}\left(I-T_{\widetilde{K}_{p}} T_{\widetilde{K}_{p}}^{*}\right) H_{G^{*}}
$$

Proof. Assume $T_{G}$ is hyponormal. By Theorem 5.1, $T\left(\widehat{K}_{p}\right)$ is a contraction. In particular $K_{0}$ is a contraction. So $K_{0} G_{M}^{*}=G_{-m}$ implies that $G_{M} G_{M}^{*} \geq G_{-m}^{*} G_{-m}$. This proves (a). It follows from

$$
\left[T_{G}^{*}, T_{G}\right]=H_{G^{*}}^{*} H_{G^{*}}-H_{G}^{*} H_{G} \leq H_{G^{*}}^{*} H_{G^{*}}
$$

that $\operatorname{rank}\left[T_{G}^{*}, T_{G}\right] \leq \operatorname{rank} H_{G^{*}}$. But the rank of $H_{G^{*}}$ is $n M$, by Lemma 2.6. Since $G-K_{p}(z) G^{*} \in H^{\infty}\left(\mathbb{C}^{n \times n}\right)$,

$$
\begin{aligned}
{\left[T_{G}^{*}, T_{G}\right] } & =H_{G^{*}}^{*} H_{G^{*}}-H_{G}^{*} H_{G}=H_{G^{*}}^{*} H_{G^{*}}-H_{K_{p} G^{*}}^{*} H_{K_{p} G^{*}} \\
& =H_{G^{*}}^{*} H_{G^{*}}-H_{G^{*}}^{*} T_{\widetilde{K}_{p}} T_{\widetilde{K}_{p}}^{*} H_{G^{*}}=H_{G^{*}}^{*}\left(I-T_{\widetilde{K}_{p}} T_{\widetilde{K}_{p}}^{*}\right) H_{G^{*}} .
\end{aligned}
$$

This is part (c). A subtle point here is that $I-T_{\widetilde{K}_{p}} T_{\widetilde{K}_{p}}^{*}$ may not be positive, but $\left[T_{G}^{*}, T_{G}\right]$ is positive. Let $K(z) \in H^{\infty}\left(\mathbb{C}^{n \times n}\right)$ be such that $\|K\|_{\infty} \leq 1$ and $G-K G^{*} \in$ $H^{\infty}\left(\mathbb{C}^{n \times n}\right)$. As in (5-3) above, $K(z)=z^{M-m} \widetilde{Y(z)}$ for some $Y(z) \in H^{\infty}\left(\mathbb{C}^{n \times n}\right)$ with $\|Y\|_{\infty} \leq 1$ and

$$
\begin{aligned}
{\left[T_{G}^{*}, T_{G}\right]=} & H_{G^{*}}^{*}\left(I-T_{\widetilde{K}} T_{\widetilde{K}}^{*}\right) H_{G^{*}}=H_{G^{*}}^{*}\left(I-T_{z^{M-m} Y(z)} T_{z^{M-m} Y(z)}^{*}\right) H_{G^{*}} \\
= & H_{G^{*}}^{*}\left(I-T_{z^{M-m} I_{n}} T_{z^{M-m} I_{n}}^{*}+T_{z^{M-m} I_{n}} T_{z^{M-m} I_{n}}^{*}-T_{z^{M-m} Y(z)} T_{z^{M-m} Y(z)}^{*}\right) H_{G^{*}} \\
= & H_{G^{*}}^{*}\left(I-T_{z^{M-m} I_{n}} T_{z^{M-m} I_{n}}^{*}\right) H_{G^{*}} \\
& \quad+H_{G^{*}}^{*}\left(T_{z^{M-m} I_{n}} T_{z^{M-m} I_{n}}^{*}-T_{z^{M-m} Y(z)} T_{z^{M-m} Y(z)}^{*}\right) H_{G^{*}},
\end{aligned}
$$

where $I_{n}$ is the $n \times n$ identity matrix. Since $\left\|T_{Y(z)}\right\| \leq 1$,

$$
\begin{aligned}
H_{G^{*}}^{*}\left(I-T_{z^{M-m} I_{n}} T_{z^{M-m} I_{n}}^{*}\right) H_{G^{*}} & =H_{G^{*}}^{*} H_{\bar{z}^{M-m} I_{n}} H_{\bar{z}^{M-m} I_{n}}^{*} H_{G^{*}}, \\
T_{z^{M-m} I_{n}} T_{z^{M-m} I_{n}}^{*}-T_{z^{M-m} Y(z)} T_{z^{M-m} Y(z)}^{*} & =T_{z^{M-m} I_{n}}\left(I-T_{Y(z)} T_{Y(z)}^{*}\right) T_{z^{M-m} I_{n}}^{*} \geq 0 .
\end{aligned}
$$

Therefore $\left[T_{G}^{*}, T_{G}\right] \geq H_{G^{*}}^{*} H_{\bar{z}^{M-m} I_{n}} H_{\bar{z}^{M-m} I_{n}}^{*} H_{G^{*}}$, and

$$
\operatorname{rank}\left[T_{G}^{*}, T_{G}\right] \geq \operatorname{rank}\left(H_{G^{*}}^{*} H_{\bar{z}^{M-m} I_{n}}\right) .
$$

By Lemma 2.6,

$$
\operatorname{rank}\left(H_{G^{*}}^{*} H_{\bar{z}^{M-m} I_{n}}\right)=\operatorname{rank}\left(H_{\bar{z}^{M-m} I_{n}}\right)=n(M-m),
$$

so $\operatorname{rank}\left[T_{G}^{*}, T_{G}\right] \geq n(M-m)$.

The next result treats the extremal case $G_{M} G_{M}^{*}=G_{-m}^{*} G_{-m}$. 
Corollary 5.3. Let $G=G_{+}+G_{0}+G_{-}^{*}=\sum_{j=-m}^{M} G_{j} e^{i j \theta}$ be a matrix trigonometric
polynomial, where

$$
G_{+}=\sum_{j=1}^{M} G_{j} e^{i j \theta} \quad \text { and } \quad G_{-}^{*}=\sum_{j=-m}^{-1} G_{j} e^{i j \theta} .
$$

Assume that $G_{M}$ is invertible and $G_{M} G_{M}^{*}=G_{-m}^{*} G_{-m}$. Then $T_{G}$ is hyponormal if and only if the following two conditions hold:

(a) $G^{*} G=G G^{*}$.

(b) There exists a constant unitary matrix $U$ such that

$$
G_{-(m-j)}=U G_{M-j}, \quad j=0,1, \ldots, m-1 .
$$

If this is the case we have

$$
\left[T_{G}^{*}, T_{G}\right]=H_{G^{*}}^{*} H_{\bar{z}^{M-m} I_{n}} H_{\bar{z}^{M-m} I_{n}}^{*} H_{G^{*}} \quad \text { and } \quad \operatorname{rank}\left[T_{G}^{*}, T_{G}\right]=n(M-m) .
$$

Proof. Assume $T_{G}$ is hyponormal. Let $U$ be the constant unitary matrix such that $U G_{M}^{*}=G_{-m}$. By (5-6), $K_{0} G_{M}^{*}=G_{-m}$; thus $K_{0}=U$. Since $T\left(\widehat{K}_{p}\right)$ defined by $(5-7)$ is a contraction, we must have $K_{i}=0$ for $i=1, \ldots, m-1$. Thus $\widehat{K}_{p}(z)=$ $K_{0}=U$ and $K_{p}(z)=z^{M-m} U$. Referring back to (5-4), $G_{-}^{*}-K_{p} G_{+}^{*} \in H^{\infty}\left(\mathbb{C}^{n \times n}\right)$ becomes

$$
\sum_{j=-m}^{-1} G_{j} e^{i j \theta}-e^{i(M-m) \theta} U \sum_{j=1}^{M} G_{j}^{*} e^{-i j \theta}=Q_{0}(z)
$$

for some $Q_{0}(z) \in H^{\infty}\left(\mathbb{C}^{n \times n}\right)$. Thus condition (b) holds. The formulas for $\left[T_{G}^{*}, T_{G}\right]$ and $\operatorname{rank}\left[T_{G}^{*}, T_{G}\right]$ follow from the proof of the previous corollary. The other direction of this result is also clear from the argument above.

Scalar versions of some results in this section are found in [Farenick and Lee 1996; Hwang and Lee 2002; Zhu 1995].

\section{References}

[Abrahamse 1976] M. B. Abrahamse, "Subnormal Toeplitz operators and functions of bounded type”, Duke Math. J. 43:3 (1976), 597-604. MR 55 \#1126 Zbl 0332.47017

[Brown and Halmos 1963] A. Brown and P. R. Halmos, "Algebraic properties of Toeplitz operators", J. Reine Angew. Math. 213 (1963), 89-102. MR 28 \#3350 Zbl 0116.32501

[Cowen 1988a] C. C. Cowen, "Hyponormality of Toeplitz operators", Proc. Amer. Math. Soc. 103:3 (1988), 809-812. MR 89f:47038 Zbl 0668.47021

[Cowen 1988b] C. C. Cowen, "Hyponormal and subnormal Toeplitz operators", pp. 155-167 in Surveys of some recent results in operator theory, vol. 1, edited by J. B. Conway and B. B. Morrel, Pitman Res. Notes Math. Ser. 171, Longman Sci. Tech., Harlow, 1988. MR 90j:47022 Zbl 0677.47017 [Farenick and Lee 1996] D. R. Farenick and W. Y. Lee, "Hyponormality and spectra of Toeplitz operators", Trans. Amer. Math. Soc. 348:10 (1996), 4153-4174. MR 97k:47027 Zbl 0862.47013 
[Farenick and Lee 1997] D. R. Farenick and W. Y. Lee, "On hyponormal Toeplitz operators with polynomial and circulant-type symbols", Integral Equations Operator Theory 29:2 (1997), 202210. MR 98j:47059 Zbl 0899.47013

[Foias and Frazho 1990] C. Foias and A. E. Frazho, The commutant lifting approach to interpolation problems, Operator Theory: Advances and Applications 44, Birkhäuser Verlag, Basel, 1990. MR 92k:47033 Zbl 0718.47010

[Gu 1994] C. X. Gu, "A generalization of Cowen's characterization of hyponormal Toeplitz operators”, J. Funct. Anal. 124:1 (1994), 135-148. MR 95j:47034 Zbl 0815.47029

[Gu and Shapiro 2001] C. Gu and J. E. Shapiro, "Kernels of Hankel operators and hyponormality of Toeplitz operators", Math. Ann. 319:3 (2001), 553-572. MR 2001m:47049 Zbl 0987.47016

[Gu and Zheng 1998] C. Gu and D. Zheng, "Products of block Toeplitz operators", Pacific J. Math. 185:1 (1998), 115-148. MR 99h:47032 Zbl 0922.47023

[Hwang and Lee 2002] I. S. Hwang and W. Y. Lee, "Hyponormality of trigonometric Toeplitz operators”, Trans. Amer. Math. Soc. 354:6 (2002), 2461-2474. MR 2003a:47057 Zbl 1011.47023

[Hwang et al. 1999] I. S. Hwang, I. H. Kim, and W. Y. Lee, "Hyponormality of Toeplitz operators with polynomial symbols", Math. Ann. 313:2 (1999), 247-261. MR 2000a:47056 Zbl 0927.47018

[Itô and Wong 1972] T. Itô and T. K. Wong, "Subnormality and quasinormality of Toeplitz operators”, Proc. Amer. Math. Soc. 34 (1972), 157-164. MR 46 \#2472 Zbl 0239.47027

[Nakazi and Takahashi 1993] T. Nakazi and K. Takahashi, "Hyponormal Toeplitz operators and extremal problems of Hardy spaces", Trans. Amer. Math. Soc. 338:2 (1993), 753-767. MR 93j:47040 Zbl 0798.47018

[Sarason 1967] D. Sarason, "Generalized interpolation in $H^{\infty}$ ", Trans. Amer. Math. Soc. 127 (1967), 179-203. MR 34 \#8193 Zbl 0145.39303

[Zhu 1995] K. H. Zhu, "Hyponormal Toeplitz operators with polynomial symbols", Integral Equations Operator Theory 21:3 (1995), 376-381. MR 95m:47044 Zbl 0845.47022

Received July 29, 2003. Revised June 23, 2005.

CAXING GU

DEPARTMENT OF MATHEMATICS

California Polytechnic State University

SAN LUIS OBISPO, CA 93407

cgu@calpoly.edu

JACOB HENDRICKS

DEPARTMENT OF MATHEMATICAL SCIENCES

UNIVERSITY OF ARKANSAS

FAYETTEVILLE, AR 72701

jghendr@uark.edu

DANIEL RUTHERFORD

DEPARTMENT OF MATHEMATICS

UNIVERSITY OF CALIFORNIA

DAVIS, CA 95616

dan_rutherford@math.ucdavis.edu 